\title{
LES TUNNELS ALPINS : OBSERVATOIRES DE L'HYDROGEOLOGIE DES GRANDS MASSIFS MONTAGNEUX
}

\author{
Jean-Christophe MARECHAL ${ }^{(1)}$ \\ ${ }^{(1)}$ Bureau de Recherches Géologiques et Minières - EAU/NRE, 1039 rue de Pinville, 34000 Montpellier, e-mail: \\ jc.marechal@brgm.fr
}

\begin{abstract}
L'étude des venues d'eau rencontrées lors de la réalisation d'ouvrages souterrains au travers des Alpes a permis de caractériser les circulations profondes d'eau souterraine au sein de ces massifs montagneux. Les principaux résultats sont illustrés au moyen de l'exemple du tunnel du Mont-Blanc. Les perméabilités mesurées dans les tunnels sont dépendantes de trois paramètres : l'échelle (forte variabilité des perméabilités dans les petits tronçons), la lithologie (roches cristallines plus perméables que les roches cristallophylliennes) et la profondeur (décroissance de perméabilité avec la profondeur du tunnel) des tronçons considérés. Une zone décomprimée caractérise les 600 premiers mètres au sein desquels la perméabilité est plus élevée. La température des eaux s'avère être un traceur efficace des circulations d'eau dans les massifs, l'eau s'infiltrant en altitude à température peu élevée contribuant au refroidissement des massifs. Les âges des eaux sont cohérents avec l'hydrodynamisme du massif, avec la présence d'eaux anciennes dans les parties les moins perméables et d'eaux récentes dans les zones les plus perméables. Un mélange d'eaux récentes et anciennes est observé dans les zones à perméabilité intermédiaire. Dans la plupart des ouvrages, les teneurs en Oxygène-18 sont compatibles avec des aires de recharge situées verticalement au droit des venues d'eau. Seules quelques exceptions mettent en évidence des circulations régionales, l'influence de la zone décomprimée ou l'infiltration d'eaux de surface (lacs).
\end{abstract}

MOTS CLEFS : hydrogéologie, perméabilité, température, roche cristalline, isotope

\section{The Mont-Blanc road tunnel: example of groundwater observatory in the Alps}

The study of water inflows in tunnels through the Alps allow characterizing deep groundwater flows within mountainous massifs. Main results are illustrated using the Mont-Blanc road tunnel example. Measured permeability into tunnels depends on three parameters: the scale (high variability of permeability in small tunnel parts), the lithology (crystalline rocks more permeable than micas-rich rocks) and the depth (permeability decrease with depth) of the studied parts of the tunnels. A "decompressed zone" corresponds to the first $600 \mathrm{~m}$ of the massif within which the permeability is higher. Water temperature is a good indicator of deep groundwater flow, water infiltrating at high altitudes being colder, then has a cooling effect on the massif. Ages of water determined using tritium are dependent on the hydrodynamics of the massif with recent water in highly permeable zones, old water in low permeability zones and a mixing of water in medium permeability zones. Oxygen-18 data primarily reflect the recharge altitude, which can be predicted a priori by considering the large-scale geological structures of each massif and the extent to which they control the subvertical paths followed by the groundwater. Anomalous 180 data may reflect local or general departures from this interpretation, including regional groundwater pathway, effect of "decompressed zone" or relationship with surface water bodies.

KEY WORDS : hydrogeology, permeability, temperature, crystalline rock, isotope

\section{INTRODUCTION}

L'observation des eaux souterraines au sein des massifs cristallins alpins est rendue difficile par les conditions d'accès, mais aussi par la présence de glaciers et couvertures quaternaires. Hormis en Suisse avec les travaux de la Société Coopérative Nationale pour l'Entreposage des Déchets Radioactifs sur les sites du Grimsel ou du Piz Pian Grand [Cedra, 1988], l'absence de forages profonds empêche d'obtenir une information directe sur les circulations d'eau souterraine dans un tel contexte. Ces roches ont fait l'objet d'études hydrogéologiques dans les grandes régions de socle comme les boucliers indien [Dewandel et al., 2006] et africain [Taylor \& Howard, 2000] dont l'histoire tectonique est marquée par l'absence d'activité récente et le développement de profils d'altération importants. Ainsi, l'hydrogéologie spécifique des massifs cristallins alpins est encore fortement méconnue.

La réalisation de longs tunnels routiers ou ferroviaires ainsi que de grandes galeries hydrauliques dans le cadre de projets hydro-électriques constitue une opportunité d'observer directement le milieu souterrain et les venues d'eau résultant des circulations souterraines. Dans cette étude, un réseau de huit ouvrages souterrains situés dans divers contextes géologiques alpins a été suivi au moyen d'un dispositif gigogne 
comprenant différents niveaux d'instrumentations : suivi en continu, suivi mensuel, suivi annuel des venues selon les ouvrages étudiés. La méthodologie employée repose sur l'étude des débits, de la chimie, des teneurs en isotopes et éléments traces des venues d'eau, mais aussi sur la réalisation d'essais de traçage et la modélisation numérique. L'objectif est de replacer les venues d'eau et leurs caractéristiques dans leur contexte géologique afin de mieux comprendre l'hydrogéologie profonde de ce type de massif. Les principaux résultats obtenus sont illustrés au travers de l'exemple du tunnel du Mont-Blanc.

\section{RESEAU D'OBSERVATION ET TUNNEL DU MONT-BLANC}

Les différentes voies de communication nord - sud au travers des Alpes ont nécessité le creusement de plusieurs ouvrages souterrains au sein des massifs cristallins. Lors du percement de ces ouvrages, s'ils ne sont pas spécialement traités, ceux-ci agissent comme un drain au travers du massif. Huit ouvrages ont été étudiés dans ce travail, situés en France, Suisse et Italie (Figure 1a). Il s'agit des tunnels routiers du MontBlanc (MB), du Gothard (GOT), du Grand-St-Bernard (GSB) et du San Bernardino (SB), de la galerie hydroélectrique de Cleuson-Dixence (DIX), du tunnel ferroviaire de la Vereina (VER) et des galeries de reconnaissance du Gothard (ATG) et de la Romanche (ROM) dont la moitié était en phase de construction durant ce travail tandis que les autres étaient en phase d'exploitation. Mis à part les galeries de la Romanche, dont la longueur n'excède pas $710 \mathrm{~m}$, ce sont de longs ouvrages dont les longueurs sont supérieures à 4000 m.

Le tunnel du Mont-Blanc (Figure 1b), perpendiculaire à l'orientation du massif, traverse celui-ci sous les Aiguilles du Midi et de Toule : sa longueur totale est de 11600 mètres. De l'entrée française (Chamonix), le tunnel recoupe sur $3100 \mathrm{~m}$ une série de schistes cristallins, puis $425 \mathrm{~m}$ de métamorphisme de contact. Il traverse ensuite la masse granitique centrale sur environ $6775 \mathrm{~m}$ avant de pénétrer dans des terrains mésozoïques essentiellement carbonatés $(1300 \mathrm{~m})$. D'importantes venues d'eau ont été observées lors du percement (Figure 1b), notamment dans une zone particulièrement fracturée située à 3000 mètres de l'entrée italienne (zone G3).

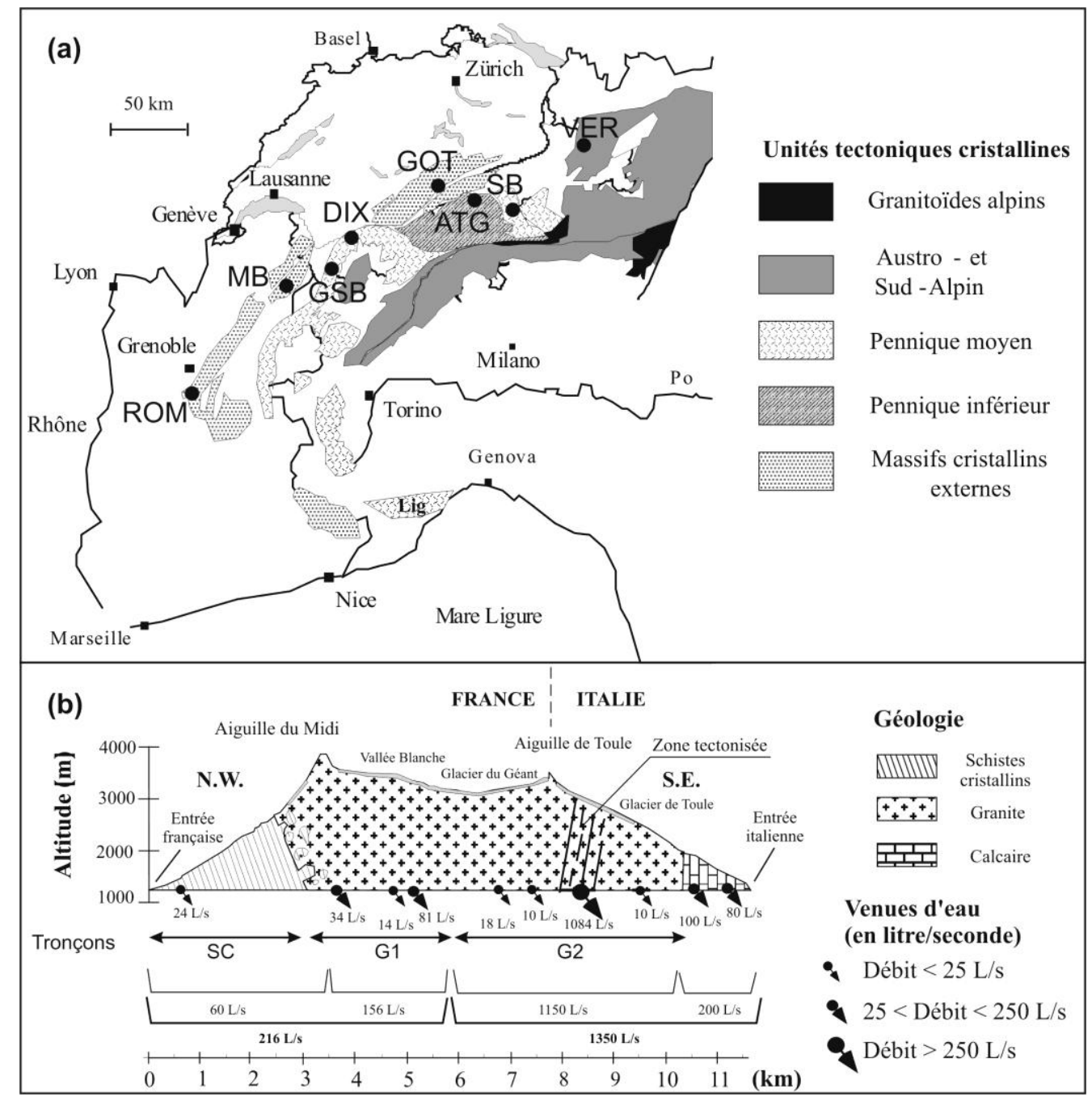


Figure 1: (a) Carte tectonique des Alpes Occidentales avec les “ ensembles cristallins " et sites observés

(b) Coupe hydrogéologique du tunnel du Mont-Blanc avec la localisation des débits initiaux des principales venues d'eau lors du percement (SC : tronçon dans les schistes cristallins ; Gi : tronçon granitiques)

\section{PERMEABILITTES}

En hydrogéologie, la perméabilité $K$ caractérise l'aptitude intrinsèque d'un milieu souterrain à être traversé par l'eau. Dans un massif montagneux fracturé, l'eau s'écoule principalement au sein du réseau de fractures dont les caractéristiques suivantes sont essentielles: orientation, extension, ouverture, remplissage, connectivité entre les fractures. Dans un tel milieu, la perméabilité varie fortement dans l'espace et en fonction de l'échelle de mesure [Clauser, 1992] : ceci traduit la forte hétérogénéité du milieu fracturé.

\section{III.1 Effet de la lithologie}

Le drainage d'un tunnel peut être assimilé à un essai de pompage à rabattement imposé et débit variable dans un forage horizontal [Maréchal \& Perrochet, 2003]. Les débits des venues d'eau mesurés au droit de leur arrivée en cours de percement ou en phase d'exploitation permettent alors de déterminer les perméabilités des massifs traversés au moyen de solutions analytiques respectivement en mode transitoire ou permanent selon que le débit a été mesuré en cours de percement et va diminuer au cours du temps ou qu'il a été mesuré après plusieurs années une fois stabilisé [Maréchal, 1998; Maréchal, 1999a]. Les solutions analytiques permettent d'ajuster le débit mesuré grâce à une perméabilité donnée en fonction d'un rabattement imposé (hauteur piézométrique de la nappe au-dessus du tunnel). Les résultats montrent (Figure 2a) que le rapport entre les perméabilités des formations les plus perméables et les moins perméables est de l'ordre de $10^{6}$; il traduit une grande variabilité de ce paramètre dans les terrains cristallins alpins. La perméabilité moyenne est proche de $10^{-8} \mathrm{~m} / \mathrm{s}$, ce qui est assez faible (à titre de comparaison, un milieu est considéré comme très perméable lorsque sa perméabilité est supérieure à $10^{-5} \mathrm{~m} / \mathrm{s}$ et imperméable lorsqu'elle est inférieure à $10^{-9} \mathrm{~m} / \mathrm{s}$ ). Il convient de rappeler qu'il s'agit de perméabilités à l'échelle du massif et non à l'échelle d'une venue d'eau particulière. Ce sont donc des valeurs moyennes sur de larges tronçons dans lesquels alternent quelques zones plus perméables (où $\mathrm{K}$ peut atteindre $10^{-5} \mathrm{~m} / \mathrm{s}$ par exemple, ou plus) au milieu de zones très peu perméables (où $\mathrm{K}$ n'excède pas par endroits $10^{-12} \mathrm{~m} / \mathrm{s}$ ). D'ailleurs, parmi les mesures effectuées à l'échelle du massif, une diminution de la variabilité de la perméabilité est observée lorsque la longueur des tronçons testés croît (Figure 2a). Les valeurs mesurées convergent vers la valeur moyenne $K=$ $9.10^{-9} \mathrm{~m} / \mathrm{s}$. Ce phénomène traduit l'hétérogénéité de la perméabilité à l'échelle du massif : alors que les petits tronçons (longueur < $1000 \mathrm{~m}$ ) peuvent correspondre à des zones locales aux caractéristiques extrêmes, les grands tronçons (longueur $>5000 \mathrm{~m}$ ) sont constitués de l'assemblage de plusieurs types de zones aux caractéristiques diverses qui conduisent à une perméabilité globale moyennée. Les résultats obtenus suggèrent également l'existence d'un comportement hydrodynamique différent selon la lithologie des terrains (Figure 2b), celle-ci étant connue grâce aux coupes géologiques des divers ouvrages [Maréchal, 1998]. Ainsi, les micaschistes du massif externe de Belledonne, les schistes cristallins (des massifs externes et des nappes simploniques) et les paragneiss (massifs externes de l'Aar et du Gothard) figurent parmi les lithologies les moins perméables; leur perméabilité est par ailleurs très variable. Les orthogneiss et granitoïdes possèdent des perméabilités légèrement supérieures aux précédents, généralement comprises entre $10^{-9} \mathrm{~m} / \mathrm{s}$ et $10^{-7} \mathrm{~m} / \mathrm{s}$. 

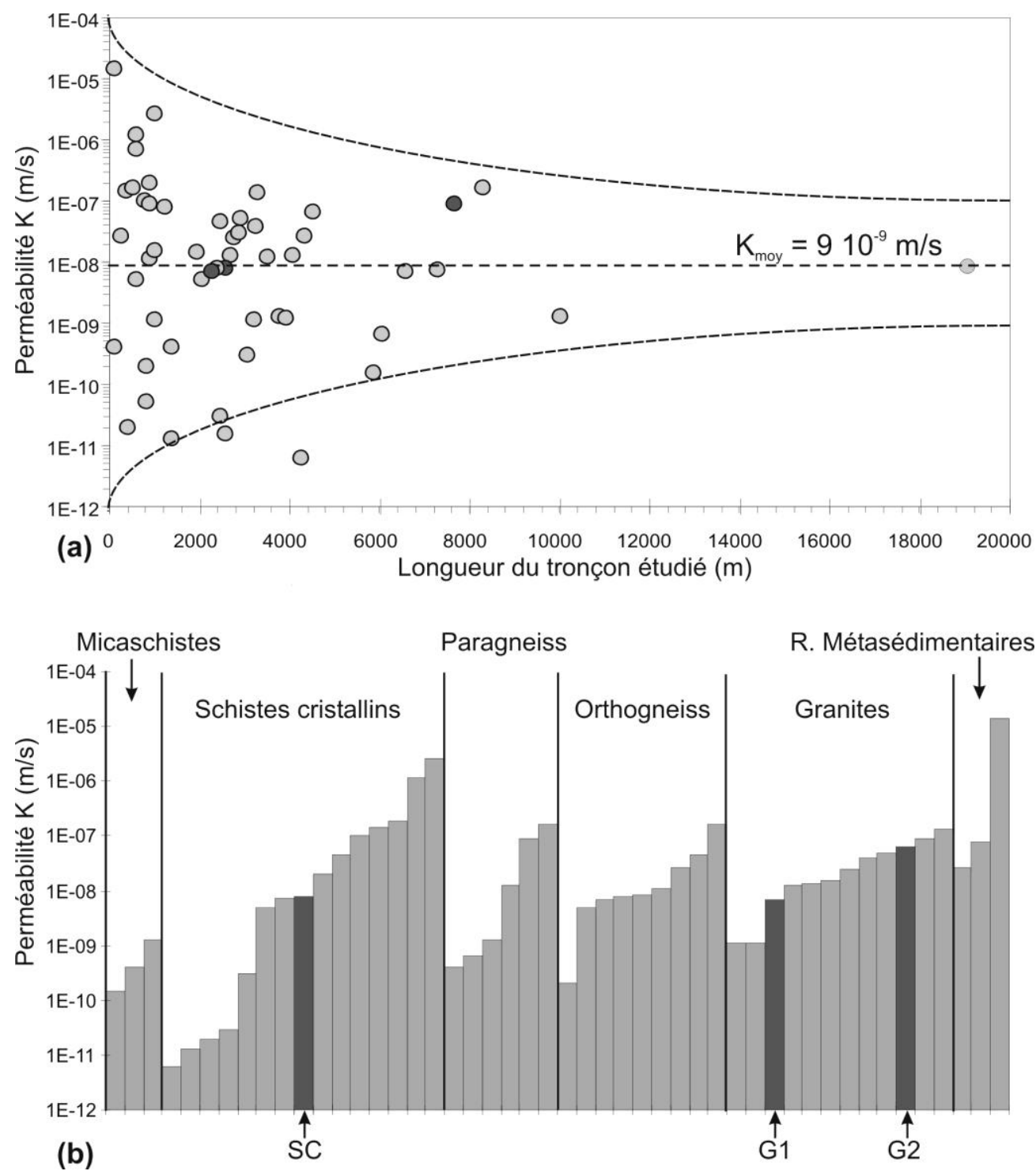

Figure 2: (a) Distribution des perméabilités calculées au moyen des solutions analytiques d'essai de pompage en fonction de la longueur des tronçons étudiés (b) Histogramme des perméabilités mesurées dans les massifs cristallins alpins, classées selon les lithologies rencontrées (les mesures au Mont-Blanc sont identifiées en gris foncé selon leur tronçon : SC, G1 et G2)

Si l'on classe les résultats en deux grands groupes de roches sur la base de leur richesse en minéraux phylliteux : les roches cristallines au sens strict (granitö̈des et orthogneiss) d'une part et les roches cristallophylliennes (schistes cristallins, micaschistes et paragneiss) d'autre part, on observe une différence de perméabilité assez nette : les roches cristallines sont six fois plus perméables en moyenne $\left(1,4.10^{-9} \mathrm{~m} / \mathrm{s}\right)$ que les roches cristallophylliennes $\left(2,2.10^{-9} \mathrm{~m} / \mathrm{s}\right)$ et environ cinquante fois plus perméables à grande profondeur (> 600 mètres). Par ailleurs, la variabilité de la perméabilité est nettement inférieure dans les roches cristallines $\left(\log K_{\max } / K_{\min }=2.9\right)$ par rapport aux roches cristallophylliennes $\left(\log K_{\max } / K_{\min }=5.6\right)$.

\section{III.2 Effet de la profondeur}

On constate, dans de nombreux ouvrages souterrains, l'existence, dans les premiers mètres de la galerie, d'une zone à plus fortes venues d'eau que dans le reste de l'ouvrage. A l'inverse, au cœur du massif, sous une épaisse couverture rocheuse, les venues d'eau sont généralement plus faibles. On citera l'exemple de la galerie Cleuson-Dixence où, pour des épaisseurs de couverture (profondeur mesurée à l'aplomb du point d'observation) supérieures à 500 mètres environ, toutes les venues d'eau possèdent des débits inférieurs à 2 L/s. Dans les zones où l'épaisseur de couverture est inférieure à 500 mètres environ, les débits oscillent le plus souvent entre 0 et $5 \mathrm{~L} / \mathrm{s}$, avec de nombreux tronçons dont le débit est supérieur à $5 \mathrm{~L} / \mathrm{s}$. Le débit maximum atteint étant de $25 \mathrm{~L} / \mathrm{s}$ sous $450 \mathrm{~m}$ de couverture environ. Les tronçons possédant les débits les plus forts se situent généralement sous de faibles épaisseurs de couverture. 
Si l'on reporte les perméabilités des roches cristallophylliennes en fonction de l'épaisseur de la couverture rocheuse au droit du point de mesure, on observe une décroissance en deux phases à tendance linéaire en diagramme semi-logarithmique (Figure 3). La décroissance de la perméabilité, suggérée par la Figure 3, peut être assimilée à une fonction exponentielle, de type :

$$
K=K_{0} e^{-\alpha C}
$$

où $K$ désigne la perméabilité $(\mathrm{m} / \mathrm{s})$ à une profondeur donnée $C(\mathrm{~m}) ; \alpha\left(\mathrm{m}^{-1}\right)$ est le taux de décroissance de la perméabilité en fonction de l'épaisseur de la couverture; $K_{0}$ est la perméabilité en surface (pour $\mathrm{C}=0$ ).

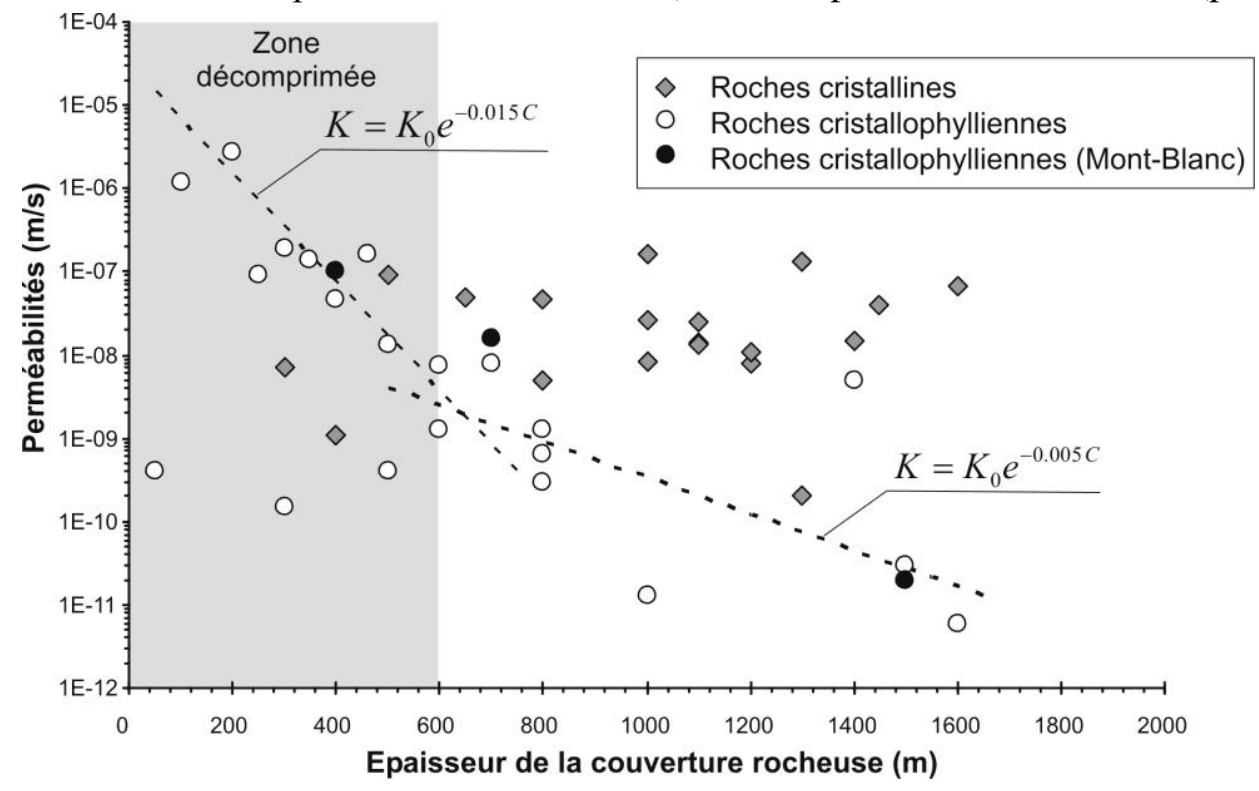

Figure 3: perméabilités mesurées en fonction de l'épaisseur de couverture rocheuse au droit des tronçons étudiés dans les huit ouvrages étudiés ; deux ajustements linéaires sont effectués sur les perméabilités des roches cristallophylliennes

Les deux ajustements de la Figure 3 sont caractérisés par deux paramètres $\alpha$ différents selon les épaisseurs de couverture rocheuse (C). Pour des épaisseurs comprises entre 100 et $600 \mathrm{~m}$ environ, le paramètre $\alpha$ vaut $0,015 \mathrm{~m}^{-1}$ tandis que pour des épaisseurs supérieures à $600 \mathrm{~m}, \alpha$ décroît à $0,005 \mathrm{~m}^{-1}$. Nous appellerons « zone décomprimée » la partie superficielle limitée à une profondeur de $600 \mathrm{~m}$. Dans ses travaux sur la zone décomprimée, Cruchet [1985] lui attribue une épaisseur voisine de 500 mètres. Les résultats obtenus par la Cedra dans le cristallin du Nord de la Suisse vont dans ce sens également (500 à 600 mètres).

La zonation proposée en fonction de la profondeur s'adresse plus spécifiquement aux roches cristallophylliennes car ces phénomènes de décompression ont été observés au cours de cette étude dans ce type de roche. C'est la forte anisotropie des roches cristallophylliennes qui peut expliquer l'importance de ces phénomènes de décompression. Dans les granitoïdes, nous nous référerons aux travaux de la Cedra qui ont mis en évidence de tels phénomènes également dans les granites et sur des épaisseurs comparables (exemples des forages de Böttstein et Siblingen [Thury et al., 1994].

L'étude des débits des venues d'eau a montré que les venues situées dans la zone décomprimée, par exemple dans les galeries de la Romanche, réagissent rapidement aux épisodes pluvieux tandis que les venues d'eau situées sous d'épaisses couvertures rocheuses sont caractérisées par des débits relativement stables comme c'est le cas dans le tunnel du Mont-Blanc [Maréchal, 1999b]. La limite de la zone décomprimée est variable et dépend des conditions locales. Il serait extrêmement dangereux de tenter de généraliser cette épaisseur à l'ensemble des Alpes.

\section{TEMPERATURES}

Hormis dans un délai court après une modification du régime de circulation d'eau souterraine, les températures des venues d'eau sont généralement en équilibre avec la roche encaissante. Les températures observées résultent d'un équilibre entre deux phénomènes aux effets opposés: d'une part, le flux géothermique réchauffe le massif rocheux et d'autre part les circulations d'eau souterraine s'infiltrant à faible température en altitude contribuent à refroidir le massif. Dès lors, les températures observées dans les tunnels constituent un bon indicateur des circulations d'eau souterraine. 
Le profil de températures observées dans le tunnel du Mont-Blanc illustre parfaitement ce phénomène (Figure 4a). Dans cet ouvrage, les températures des venues d'eau [Gudefin, 1967] sont comprises entre $8.5^{\circ} \mathrm{C}$ à 280 mètres et $33.5^{\circ} \mathrm{C}$ à 3350 mètres de l'entrée nord. Une anomalie négative (température minimale égale à $11.5^{\circ} \mathrm{C}$ ) est présente dans la zone à fortes venues d'eau (à peu près $1000 \mathrm{~L} / \mathrm{s}$, [Maréchal, 2000]) sous la Pointe Helbronner (Figure 4a). Un essai de traçage a démontré que cette venue d'eau était en relation directe avec les eaux issues de la fonte du glacier de Toule [Maréchal, 2000]. Sur le diagramme des températures mesurées à l'avancement en fonction de l'épaisseur de la couverture (Figure 4b), l'anomalie thermique apparait clairement. Deux gradients thermiques des eaux différents peuvent être déterminés. Un premier gradient $\left(\mathrm{G}_{1}=0.016^{\circ} \mathrm{C} / \mathrm{m}\right)$ caractérise les venues d'eau pour lesquelles la couverture est inférieure à $1000 \mathrm{~m}$ dans les schistes cristallins. Le second gradient thermique $\left(\mathrm{G}_{2}=0.007^{\circ} \mathrm{C} / \mathrm{m}\right)$, moins élevé, caractérise les venues d'eau situées au cœur du massif dans le granite. Une modélisation mathématique des écoulements souterrains et des échanges de chaleur dans le massif [Maréchal et al., 1999] a montré que c'est la présence de la zone fortement fracturée à grosses venues d'eau qui justifie cet écart de gradient.

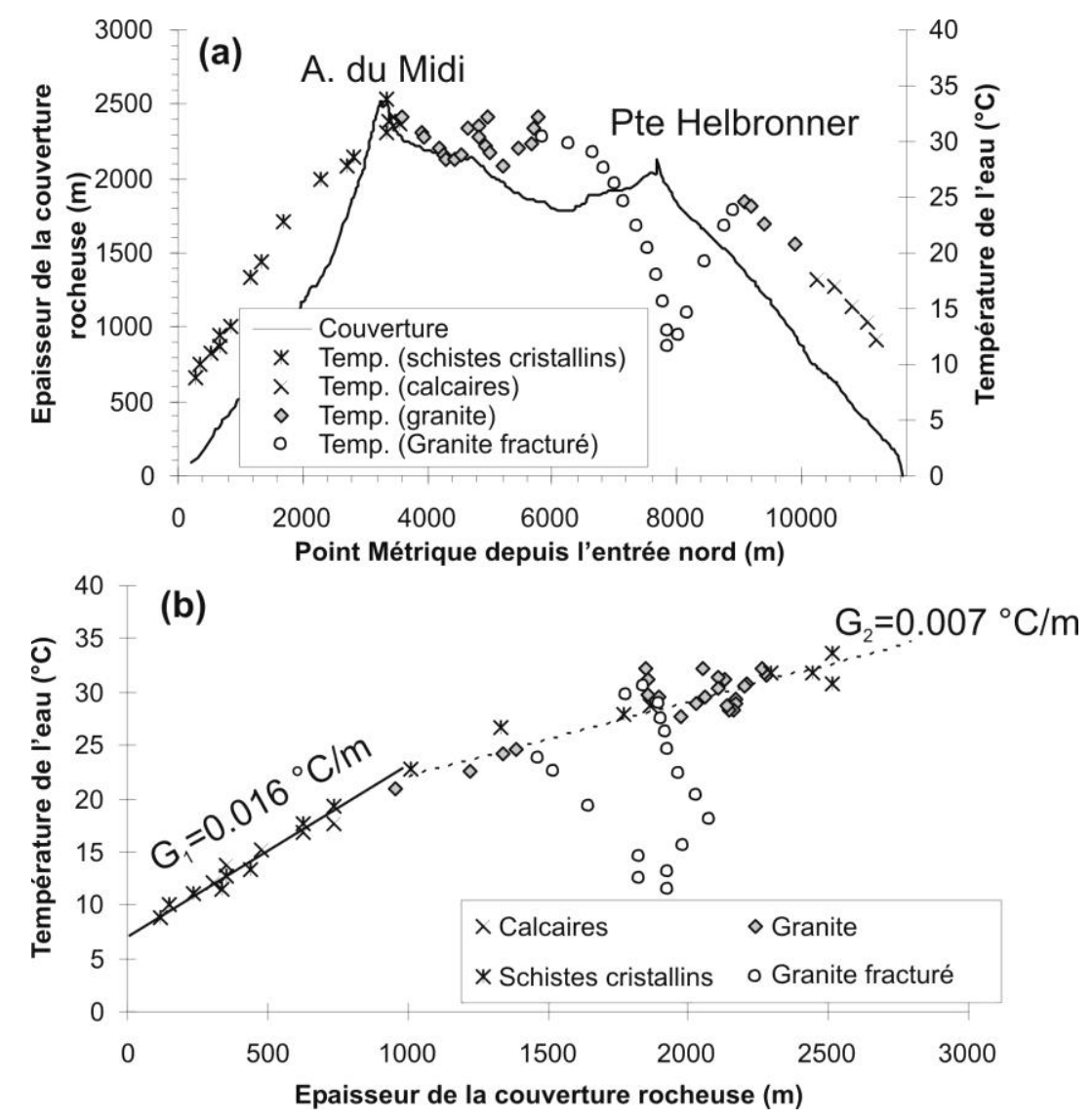

Figure 4: (a) Profil de la température des venues d'eau et de l'épaisseur de la couverture rocheuse dans le tunnel du Mont-Blanc (b) Evolution de la température de l'eau en fonction de l'épaisseur de la couverture rocheuse $\left(G_{1}\right.$ : gradient hydrothermique dans les schistes cristallins ; $G_{2}:$ gradient hydrothermique dans le granite hormis la zone de granite fracturé)

La chute de température, mesurée durant l'avancement plus de 1000 mètres avant la rencontre de la zone fortement aquifère, avait indiqué aux foreurs italiens l'imminence d'importantes venues d'eau. La même modélisation mathématique citée ci-dessus [Maréchal et al., 1999] a permis de reconstituer cette anomalie thermique et de montrer qu'il est possible, en cours d'avancement, à partir de données de température, d'améliorer quantitativement la prévision des venues d'eau dans un ouvrage souterrain [Maréchal et al., 1999]. Cette modélisation a également montré la grande inertie des phénomènes thermiques à l'échelle d'un massif montagneux, avec des températures actuelles influencées par la dernière période glaciaire il y a 10000 ans, période durant laquelle la domination des glaciers à base froide réduisait le taux d'infiltration d'eau froide et augmentait conséquemment la température souterraine [Maréchal et al., 1999].

Si l'on compare plusieurs ouvrages souterrains alpins, on observe que les gradients thermiques des eaux varient d'un ouvrage à l'autre en fonction de la quantité d'eau drainée par celui-ci. Le débit linéaire (débit total des venues d'eau dans un tronçon rapporté à sa longueur, exprimé en $1 / \mathrm{s} / \mathrm{m}$ ) et le gradient thermique possèdent une corrélation négative, illustrée sur la Figure 5. Plus il y a d'eau atteignant le tunnel en cours de 
percement, et plus il y avait donc de potentialité d'écoulement d'eau dans le massif avant le percement de l'ouvrage, plus le gradient thermique mesuré est faible. C'est l'effet réfrigérant des circulations d'eau. Quand le débit linéaire devient négligeable (de l'ordre de $10^{-4} \mathrm{l} / \mathrm{s} / \mathrm{m}$ ), le gradient thermique des eaux tend vers une valeur de $0.026{ }^{\circ} \mathrm{C} / \mathrm{m}$, valeur légèrement inférieure, suite au non confinement des massifs étudiés, au gradient géothermique mondial $\left(\mathrm{G}=0.030^{\circ} \mathrm{C} / \mathrm{m}\right)$. Sur ce diagramme, le débit linéaire dans la galerie du Gothard Alptransit (ATG) apparaît hors corrélation : des circulations d'eau non rencontrées par l'ouvrage pourraient exister dans le massif.

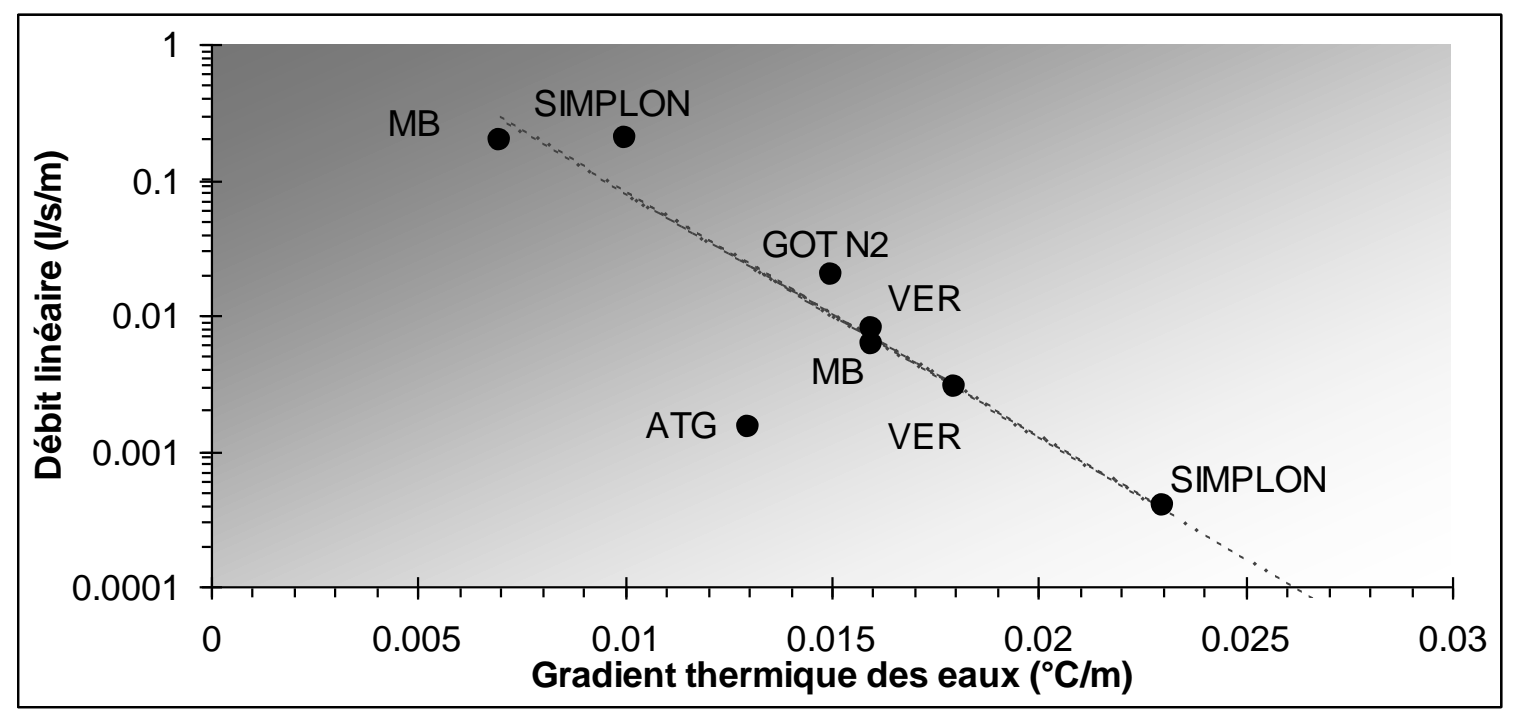

Figure 5: Relation entre le débit linéaire et le gradient thermique des eaux dans divers ouvrages souterrains (MB : Mont-Blanc ; GOT-N2 : Gothard-N2 ; VER : VEREINA ; ATG : Gothard Alptransit).

\section{AGES DES EAUX ET ALTITUDES D'INFILTRATION}

Dans ces massifs montagneux, les systèmes d'écoulement entre des zones d'alimentation situées à haute altitude (jusqu'à $3500 \mathrm{~m}$ ) et des exutoires à moins de $1500 \mathrm{~m}$ d'altitude sont longs. De plus, les faibles perméabilités d'ensemble font que les temps de transit sont élevés : l'emploi du tritium est donc un outil utile pour déterminer les temps de transit moyens des eaux souterraines. La technique repose sur la connaissance précise des intrants en tritium apportés dans l'atmosphère par les essais nucléaires des années 60 . Trentedeux venues d'eau ont fait l'objet de 53 analyses de teneur en tritium. L'application du modèle isotopique PISTON-FLOW adapté aux venues d'eau en tunnel si la dispersion hydrodynamique est négligée [Etcheverry \& Perrochet, 1999] , montre que les teneurs inférieures à 10 UT (UT : Unité Tritium, unité de mesure de la teneur en tritium d'une eau) sont caractéristiques d'eaux dont le temps de transit moyen est supérieur à 40 années : ce sont des eaux très anciennes. La Figure 6 met en relation la teneur en tritium mesurée des venues d'eau étudiées avec la perméabilité à l'échelle du massif dans le tronçon auquel elles appartiennent.

Toutes les venues d'eau situées dans des tronçons dont la perméabilité est inférieure à $10^{-9} \mathrm{~m} / \mathrm{s}$ sont des eaux très anciennes dont le temps de transit moyen est supérieur à 40 ans (hormis une venue à 30 UT, caractéristique d'un temps de transit compris entre 20 et 40 ans). Les eaux situées dans des tronçons à perméabilité supérieure à $3.10^{-7} \mathrm{~m} / \mathrm{s}$ sont récentes (teneurs entre 10 UT et 20 UT : temps moyens de transit inférieurs à 15 - 20 ans). Ainsi, un traceur fluorescent injecté dans la rimaye du glacier de Toule à l'aplomb de la zone fracturée a été retrouvé quelques semaines plus tard deux mille mètres plus bas dans le tunnel [Maréchal, 2000]. Entre ces deux zones de perméabilité, il existe une grande zone de recouvrement (entre 10${ }^{9}$ et $3.10^{-7} \mathrm{~m} / \mathrm{s}$ ) dans laquelle on trouve aussi bien des eaux récentes que très anciennes. Le manque de clarté de la relation entre les temps de transit de certaines venues et la perméabilité du massif est due à l'hétérogénéité du massif. Une venue correspond par définition à une structure (fracture ou autre) dans laquelle la perméabilité est localement plus élevée et est caractérisée par conséquent par des temps de transit plus faibles. 


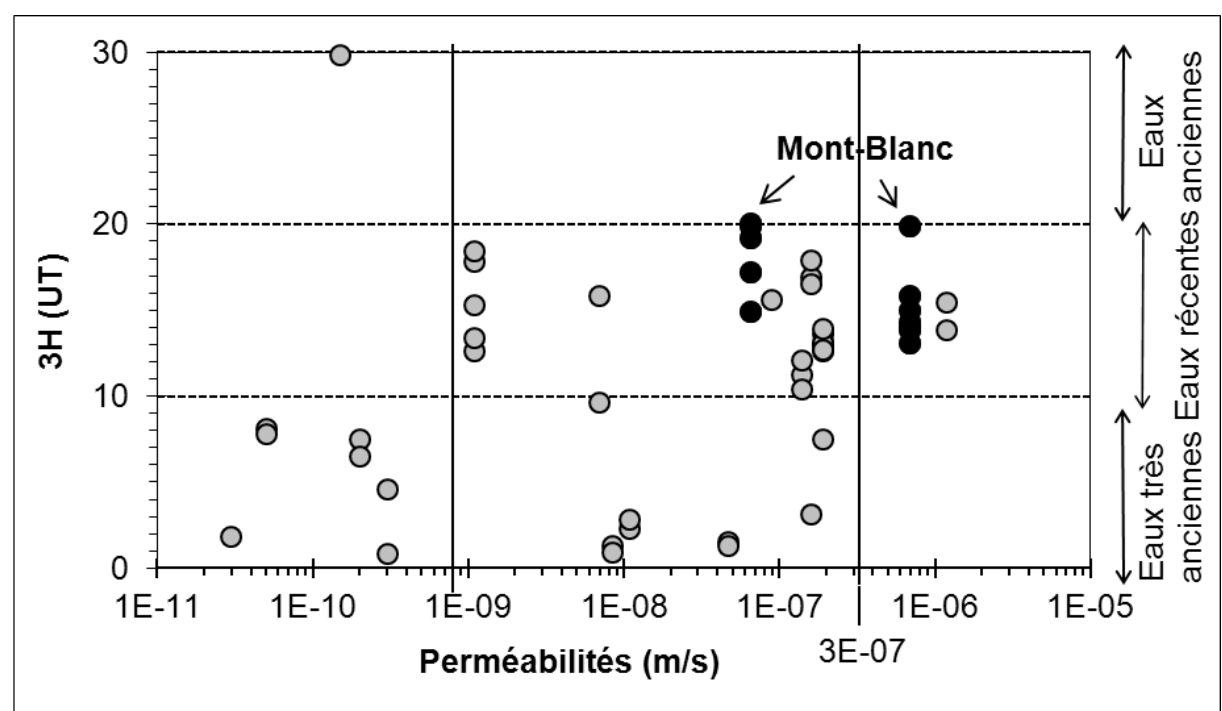

Figure 6: Teneur en tritium des venues d'eau en fonction de la perméabilité de la zone à laquelle elles appartiennent.

La vocation des ouvrages souterrains étudiés étant de traverser les massifs alpins, il en résulte que la plupart d'entre eux traversent les structures géologiques. Celles-ci possèdent un pendage souvent très redressé et une orientation perpendiculaire à la direction de l'ouvrage. Cet état de fait, combiné avec l'existence d'importants gradients hydrauliques existant entre les zones d'alimentation et l'exutoire que constitue l'ouvrage, induit des circulations d'eau globalement très redressées voire subverticales le long des structures géologiques alpines. Les altitudes potentielles d'infiltration ont été calculées en faisant l'hypothèse que les eaux circulaient dans le massif le long des structures majeures (fractures aquifères, failles, contacts tectoniques ou schistosité principale). Ces altitudes d'infiltration ont été comparées aux teneurs en oxygène18 des eaux qui sont théoriquement fonction de leur altitude d'infiltration selon un gradient d'altitude défini régionalement [Clark \& Fritz, 1997]. Sur le diagramme de la Figure 7, les altitudes potentielles d'infiltration sont mises en relation avec les teneurs en oxygène- 18.

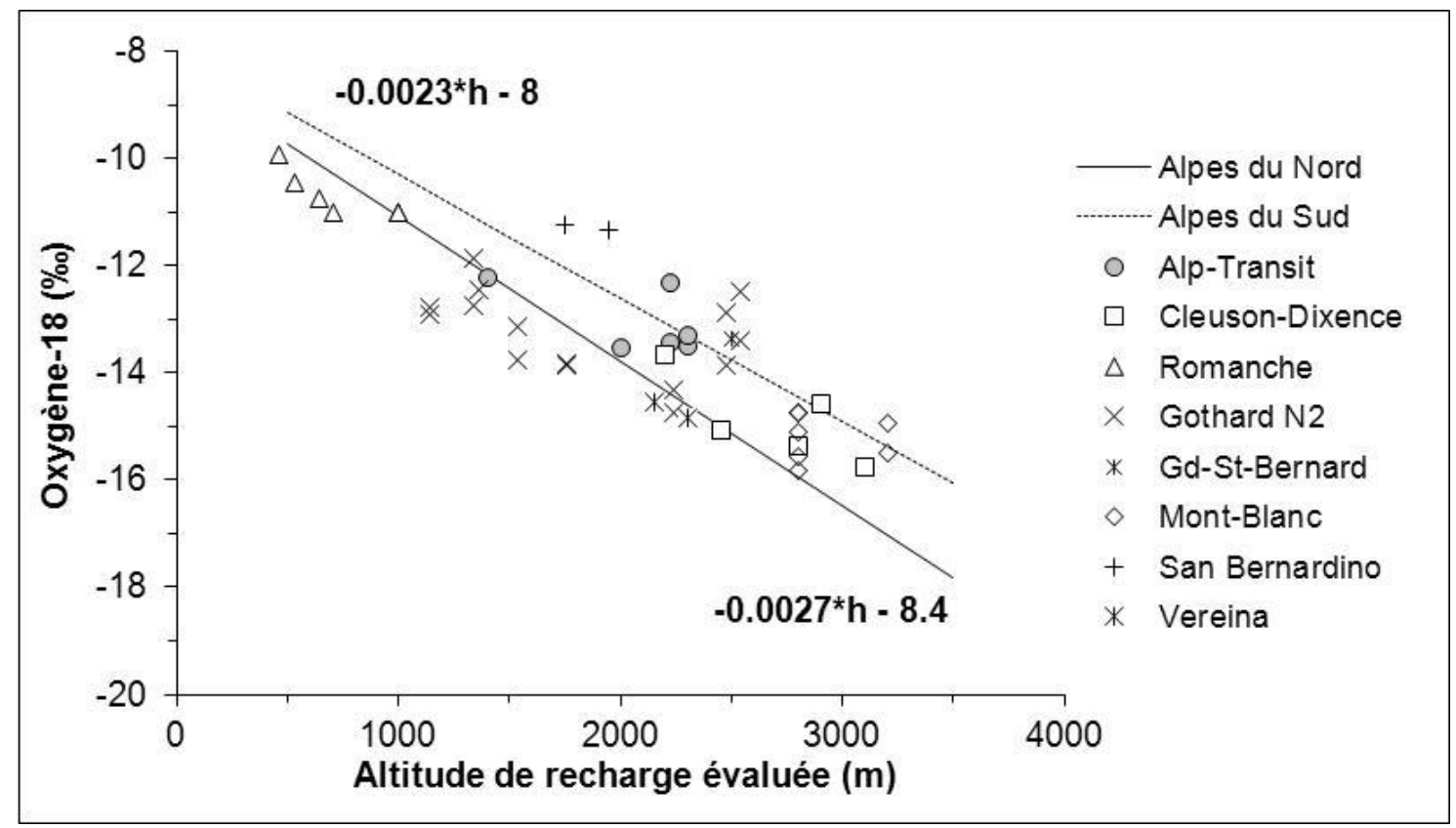

Figure 7: Diagramme oxygène-18 - altitude d'infiltration de l'eau.

A première vue, dans la plupart des cas, les points s'alignent selon les droites représentatives des précipitations dans les Alpes [Novel, 1995]. Ainsi, les tunnels du Mont-Blanc (versant sud), du Grand-SaintBernard (versant sud) et du San Bernardino (versant sud) possèdent des points qui s'alignent plutôt avec la droite des Alpes du sud. A l'inverse, les points représentatifs des autres ouvrages s'alignent plutôt selon la droite de précipitations du nord des Alpes. Ceci valide le modèle simple d'écoulement des eaux le long des structures subverticales. Les points qui s'éloignent de ces droites ont permis de mettre en évidence des exemples de circulation d'eau qui s'éloignent du modèle proposé ci-dessus, soit dans la zone décomprimée 
du massif, soit par une alimentation par des eaux de surface ou par des systèmes d'écoulements régionaux respectivement dans la galerie Alptransit Gothard et le tunnel routier du Gothard [Maréchal et Etcheverry, 2003].

\section{CONCLUSIONS}

Les ouvrages souterrains constituent des observatoires uniques des circulations d'eau souterraine en profondeur au sein des massifs cristallins alpins. Ils permettent de caractériser la perméabilité des terrains traversés à l'échelle du massif en fonction de la profondeur, la qualité chimique des eaux, leur âge moyen ainsi que leur origine. Ils démontrent la spécificité du milieu montagneux par rapport au bouclier de socle.

Ces résultats démontrent l'intérêt d'un suivi détaillé des venues d'eau lors du percement des tunnels et ce, non seulement pour le génie civil dans la perspective de la réalisation de futurs tunnels mais aussi pour les hydrogéologues dans celle d'une meilleure connaissance de ces hydrosystèmes complexes. Ces résultats apportent également des éléments de connaissance pour mieux contraindre les modèles mathématiques. A plus long terme, étant donné la forte réactivité de certaines venues d'eau aux conditions climatiques, leur suivi permettrait de mesurer l'impact des changements climatiques sur le régime d'écoulement des eaux souterraines profondes, dont la dynamique se distingue de celle des eaux alimentant les sources de montagne par exemple.

\section{REMERCIEMENTS}

Les principaux résultats exposés dans cet article ont été acquis durant un travail de thèse réalisé à l'Ecole Polytechnique Fédérale de Lausanne (thèse EPFL n 1769) et en partie financé par la Fondation EVIAN.

\section{REFERENCES ET CITATIONS}

Clark, I., Fritz, P., (1997). - Environmental Isotopes in Hydrogeology. Lewis Publishers, New-York.

Clauser, C. (1992) - Permeability of Crystalline Rocks, Eos, Transactions, American Geophysical Union, 73(21), 233-240.

Cedra (1988). - Berichterstattung über die untersuchungen der phase I am potentiellen standort Piz Pian Grand (Gemeinden Mesocco und Rossa, GR). Nagra Technischer Bericht 88-19.

Cruchet, M. (1985). - Influence de la décompression sur le comportement hydrogéologique des massifs cristallins en Basse Maurienne (Savoie, France). Géologie Alpine, 61, 65-73.

Dewandel, B., Lachassagne, P., Wyns, R., Maréchal, J.C. \& Krishnamurthy, N.S. (2006). - A generalized 3$\mathrm{D}$ geological and hydrogeological conceptual model of granite aquifers controlled by single or multiphase weathering. Journal of Hydrology 330(1-2): 260-284, doi:10.1016/j.jhydrol.2006.03.026.

Etcheverry, D. \& Perrochet, P. (1999). - Reservoir theory, groundwater transit time distributions, and lumped parameter models. In: Proc. Internat. Symp. Isotope Techniques in Water Resources Development and Management, Vienna. International Atomic Energy Agency.

Gudefin, H. (1967). - Observations sur les venues d'eau au cours du percement du tunnel sous le Mont-Blanc. Bull. B.R.G.M., 4, 95-107.

Maréchal, J.C. (1998). - Les circulations d'eau dans les massifs cristallins alpins et leurs relations avec les ouvrages souterrains. Thèse 1769. Ecole Polytechnique Fédérale de Lausanne, Suisse, 296 p.

Maréchal, J.C. (1999a). - Observation des massifs cristallins alpins au travers des ouvrages souterrains - 1. Caractérisation de la conductivité hydraulique à l'échelle du massif. Hydrogéologie, 1, 21-32.

Maréchal, J.C. (1999b). - Observation des massifs cristallins alpins au travers des ouvrages souterrains. 2. Définition du rôle hydrogéologique de la zone décomprimée. Hydrogéologie, 1, 33-42.

Maréchal, J.C. (2000). - Massif du Mont-Blanc : identification d'une structure hydrogéologique majeure. La Houille Blanche, 6, 78-86.

Maréchal, J.C., Perrochet, P. \& Tacher, L. (1999). - Long-term simulations of thermal and hydraulic characteristics in a mountain massif: the Mont-Blanc case study. Hydrogeology Journal, 7(4), 341-354.

Maréchal, J.C. \& Etcheverry, D. (2003). - The use of 3H and delta18O tracers to characterize water inflows in alpine tunnels, Applied Geochemistry, 18/3, 339-351.

Maréchal, J.C., Perrochet, P. (2003). - Nouvelle solution analytique pour l'étude de l'interaction hydraulique entre les tunnels alpins et les eaux souterraines, Bull. Soc. géol. Fr., 174/5, 441-448.

Novel, J.P. (1995) - Contribution de la Géochimie à l'étude d'un aquifère alluvial de Montagne - Cas de la vallée d'Aoste. Mem. Sci. Terre, Acad. Paris, Univ. ParisVI. 
Taylor, R., Howard, K. (2000). - A tectono-geomorphic model of the hydrogeology of deeply weathered crystalline rock: evidence from Uganda. Hydrogeology Journal 8(3), 279-294.

Thury, M., Gautschi, A., Mazurek, M., Müller, W.H., Naef, H., Pearson, F.J, Vomvoris, S., Wilson, W. (1994). - Geology and hydrogeology of the crystalline basement of Northern Switzerland. Nagra Technical Report 93-01. 